\title{
40 ans de discours sur l'enseignement de l'oral : la didactique face à ses questions
}

40 Years of Discourses on Teaching Spoken Language: Didactics Faces Its

Questions

\section{Élisabeth Nonnon}

\section{OpenEdition}

\section{Journals}

Édition électronique

URL : http://journals.openedition.org/pratiques/3115

DOI : $10.4000 /$ pratiques.3115

ISSN : 2425-2042

Éditeur

Centre de recherche sur les médiations (CREM)

\section{Référence électronique}

Élisabeth Nonnon, « 40 ans de discours sur l'enseignement de l'oral : la didactique face à ses questions », Pratiques [En ligne], 169-170 | 2016, mis en ligne le 30 juin 2016, consulté le 10 décembre 2020. URL : http://journals.openedition.org/pratiques/3115; DOI : https://doi.org/10.4000/pratiques. 3115

Ce document a été généré automatiquement le 10 décembre 2020.

(c) Tous droits réservés 


\title{
40 ans de discours sur l'enseignement de l'oral : la didactique face à ses questions
}

\author{
40 Years of Discourses on Teaching Spoken Language: Didactics Faces Its \\ Questions
}

Élisabeth Nonnon

1 Ne pouvant faire ici une carte complète de 40 ans de discours sur l'enseignement de l'oral, la présentation sera forcément stylisée et allusive, incomplète, voire partiale. Pourtant un regard en arrière est nécessaire pour reconnaitre les filiations, avancées, dilemmes et chantiers toujours ouverts. La capitalisation des acquis est difficile en didactique, et c'est particulièrement vrai pour l'oral. Je m'appuie sur une note de synthèse pour la Revue française de pédagogie en 1999, sans en avoir le souci d'exhaustivité, et pour les évolutions sur un essai de synthèse dans Pratiques en 2011. Le bilan de 1999 recensait de nombreux apports nouveaux à cette période, que je ne reprendrai qu'à très gros traits. Il y a-t-il depuis des acquis nouveaux, des points de consensus? Quels obstacles persistent, quelles catégories ou fractures continuent à structurer le champ? Quels problèmes restent ouverts, ou se sont révélés? Quels effets dans les pratiques scolaires?

2 Ces évolutions et ces points d'achoppement débordent la question de l'oral. Bien que restant un objet assez marginal et problématique en didactique du français, l'enseignement de l'oral lui pose des questions qui valent aussi pour l'écriture ou d'autres domaines. Comme l'écrivaient J. Mouchon \& F. Fillol (1977) dans l'éditorial d'un des premiers numéros de Pratiques, « au travers de toutes les discussions sur l'oral à l'école affleure le problème fondamental du contenu de l'enseignement du français ». C'est une sorte de révélateur pour la didactique, dont il met à jour les tensions et qu'il interroge dans ses choix, ses évolutions et ses orientations idéologiques, méthodologiques et épistémologiques. 


\section{Une question préalable : pourquoi est-ce si difficile pour la didactique de s'investir durablement dans l'enseignement de l'oral ?}

En 1999, je traitais de l'oral comme d'un objet paradoxal, en raison du contraste entre son importance soulignée par l'opinion, les enseignants, relayée périodiquement par l'institution (qui invoque son urgence dans la lutte contre l'échec scolaire, pour l'insertion sociale et professionnelle), et des apports didactiques plutôt hésitants, un faible investissement par les chercheurs. Cela n'a plus été tout à fait vrai pendant un moment, mais il semble que cela le soit redevenu dans la dernière période. La question de l'oral partage un certain nombre de ces caractéristiques avec celle du vocabulaire: ce sont de objets difficiles, peu confortables pour la didactique et, de ce fait, pendant longtemps peu investis par elle.

Cela tient en partie aux caractéristiques de l'objet. Depuis toujours, on évoque la diversité des acceptions que le terme recouvre, la difficulté d'en stabiliser une définition, de l'isoler d'attitudes, d'habitus sociaux, d'apprentissages cognitifs plus généraux. Cela tient aussi à ce qu'est la recherche didactique, à ses orientations récentes, ses conditions d'exercice, ses limites. Elle a du mal à affronter ces objets qui la débordent, échappent à une définition simple de la transposition didactique (restée prégnante quoi qu'on dise). J.-F. Halté écrivait que la maitrise de l'écrit s'enseigne difficilement, parce qu'elle excède les limites du cours de français, mais c'est encore plus vrai de l'oral. Il maintient ouverte la question posée par J.-F. Halté $(1992,2006)$ des rapports entre l'enseignable et l'apprenable : il peut être un objet de travail explicite à l'école, cela ne veut pas forcément dire qu'il s'enseigne. Comme disait J.-L. Chiss en 2002, il pose la question de la didactique sur une autre base que celle de la didactisation de savoirs, et ce n'est pas facile pour elle. Cela questionne donc le statut et la fonction des savoirs que veut construire la recherche, leur rapport à la pratique scolaire, la distinction entre ceux à transposer pour l'élève et ceux qui seraient des outils pour l'enseignant. Cet écart questionne l'investissement de la didactique face à des domaines reconnus centraux pour la lutte contre l'échec scolaire mais complexes, sujets aux variations sociales et posant le problème de la norme, résistant à une transposition didactique simple, de plus dont l'étude est couteuse pour la recherche comme pour la pratique enseignante.

5 Pour évoquer évolutions, acquis et dilemmes, je reprendrai ces quatre points : la question de l'échec scolaire et de la dimension sociale; les savoirs sur l'objet et leur rapport avec l'entreprise didactique; les tâtonnements autour de l'enseignabilité ; le rapport de la didactique à l'analyse du travail et à l'efficience.

\section{Les contextes de mobilisation de la question de l'oral et ses enjeux politiques}

6 Les difficultés font qu'on peut encore parler d'une histoire à éclipses, avançant de façon spiralaire avec reprise des mêmes questions, plutôt que de progrès linéaire dans la recherche et les transformations de l'enseignement. Il y a des moments d'investissement, puis des apories, des difficultés théoriques et pratiques qui découragent de poursuivre, la question passe au second plan ou se dilue, puis réapparait périodiquement. En témoigne 
sa présence en pointillés au fil des numéros de Pratiques. Elle est visible dans les années 1976, 1977, 1980, 1983, soit dans des numéros qui lui sont dédiés ( $n^{\circ} 17$ : «L'oral » en $1977 ; n^{\circ} 40$ : « La communication » en 83), soit dans des articles dans des numéros où un type de discours est étudié à l'oral comme à l'écrit (récit oral plusieurs fois, deux articles importants sur l'argumentation à l'oral dans le n 28, «Argumenter » en 1980 - François ; Garcia); puis un long silence, des numéros comme "Argumentation et langue ", « Enseigner l'argumentation », « Polyphonie », « Le récit »... n'intégrant pas les pratiques orales, dialogues et façons de parler étant abordés sous leur forme littéraire, romanesque ou théâtrale. La question réapparait vers les années 90 sous l'impulsion de J.-F Halté, en termes d'interactions (1993; $\mathrm{n}^{\circ}$ 103-104, "Interactions et apprentissages »; une table ronde sur l'oral au lycée dans le n 107-108 - Denizot et al., 2000 -, deux articles dans le n - 115-116 sur "L'écriture et son apprentissage » en 2002 - Nonnon; Delcambre), puis repart à l'arrière-plan, avec quelques réapparitions ( $n^{\circ} 147-148$, méthodologique, sur "Interactions et corpus oraux », et deux articles en 2011 dans un bilan sur la didactique, $\mathrm{n}^{\circ}$ 149-150 - Nonnon ; Cicurel). Les difficultés expliquent en partie les éclipses, qu'en est-il des retours de la question? Quelles évolutions dans la façon dont elle se pose, en fonction des contextes?

7 Je peux redire ce que j'écrivais en 1999 : les contextes d'émergence sont toujours chargés d'enjeux politiques et idéologiques, même quand le discours militant change ou s'estompe. On a souvent souligné que l'inquiétude orale de notre système d'enseignement était ancienne et périodique. Selon J.-C. Chevalier, la question ressurgit chaque fois qu'une nouvelle population doit être intégrée, difficilement, à notre système scolaire. Cette dimension s'est dite explicitement, mais différemment, à deux périodes clés.

8 Les années 70-80 - période des débuts de Pratiques - découvrent l'importance de l'échec scolaire, des facteurs culturels et sociaux qui sous-tendent les inégalités. La question de la maitrise du langage est posée de façon politique, comme inséparable de la mission démocratisante de l'école. Cette période voit nombre d'études sur le rôle des difficultés langagières dans l'échec scolaire. Les travaux sur l'oral ont été pour beaucoup le fait d'associations, d'équipes universitaires militantes ${ }^{1}$. Tout en s'inscrivant contre l'idéologie du handicap sociolinguistique et sa vision mécaniste du lien entre limitation cognitive et façons de parler, elles voulaient approfondir ces questions. Le projet scientifique est donc chargé sur le plan axiologique: réhabiliter le langage des exclus, affiner les lieux de difficulté linguistique réelle et non sur-normative, centrer l'attention sur les mécanismes de discrimination scolaire, et pour Pratiques, légitimer des genres, des formes de discours non légitimes. Pratiques n ${ }^{\circ} 17$, avec des articles de D. François et de C. Bachmann (1977), se situe dans cette visée. Cette époque a tenté des approches comparatives quantitatives (entre élèves de milieux différents, entre productions orales dans des tâches ou des moments différents d'une activité), selon des critères linguistiques, cherchant en quoi ils seraient indicatifs de processus cognitifs. Ces critères se révélant discutables vue la complexification des savoirs en psycholinguistique (non-correspondance entre subordination et complexité psycholinguistique par exemple) ou en pragmatique, des équipes (Paris $\mathrm{V}$ entre autres) se sont engagées dans la recherche de critères plus pertinents centrés sur la langue, ses variations hiérarchisables ou non, les possibles indicateurs linguistiques de blocages cognitifs. L'attention s'est alors déplacée de la langue aux conduites langagières (Pratiques parle alors de communication, cf. Halté, 1983) où s'actualisent les formes linguistiques et aux dialogues; l'exploration de ces fonctionnements a nécessité des analyses qualitatives sur de plus petits corpus ${ }^{2}$. Cette 
tendance a marqué la période suivante, même si les enjeux ne sont plus les mêmes : il y a eu en didactique peu d'études de corpus importants, beaucoup d'approches qualitatives sur de très petits corpus.

9 Ce contexte est le creuset où se sont formés beaucoup de parcours de recherche qui ont marqué le champ de l'oral les années suivantes, initiant des thématiques qui se poursuivent actuellement, sans forcément garder la préoccupation sociologique et militante du départ.

Dans les années 2000, le retour au premier plan correspond au discours sur la fracture sociale, l'éclatement de la culture commune: l'école et la parole partagée ont pour mission de restaurer la communauté et le partage des valeurs au-delà des clivages sociaux. Cette période est marquée par la diffusion de travaux sur l'argumentation dans la perspective de C. Perelman et J. Habermas qui assignent à l'argumentation dans l'espace public ce rôle de régulation sociale. Après une éclipse, on retrouve dans la crise actuelle ce discours liant l'oral à l'acculturation à travers les échanges conversationnels (partage littéraire, patrimonial et citoyen). On voit combien ces enjeux diffèrent de ceux de la période précédente, sur la genèse des discriminations, la conception de l'espace social de la classe et les tensions qui le traversent.

11 La période récente a aussi repris un chantier ouvert à cette époque dans le prolongement des questions de l'efficacité cognitive des conduites langagières. Intégrant la diffusion de travaux socioconstructivistes, et linguistiques sur la coénonciation, les recherches des années 90 cherchaient à articuler l'espace public de débat et des enjeux d'acquisition de connaissances, se centrant sur les indices linguistiques et pragmatiques d'une construction coopérative de significations et les déplacements sémantiques dans le dialogue ${ }^{3}$. Les études de l'argumentation lors de séances d'apprentissage, autrefois minoritaires (Langue française $n^{\circ} 112,1999$; Halté, 1993), se sont multipliées, centrées sur les indices d'une avancée commune et les régulations de l'enseignant, pour voir comment se construit un collectif de travail, une "communauté» engagée dans la même dynamique cognitive ou le partage d'un patrimoine ${ }^{4}$. Deux tendances se sont accentuées : la perspective didactique précisément centrée sur le langage s'est estompée dans une approche interdisciplinaire. Surtout, l'attention aux facteurs de discrimination que révèle l'analyse fine de discussions est devenue minoritaire, certaines références ont disparu (J. J. Gumperz par exemple), ainsi que la mention de la classe comme réalité hétérogène où la parole est inégalement distribuée et la participation différenciée à l'activité. L'hétérogénéité des individus ou des sous-groupes composant une classe interdirait pourtant d'appliquer de façon naïve la notion sociolinguistique de communauté discursive à ce collectif, tant qu'on n'a pas problématisé l'adhésion de tous au jeu scolaire en cours et le nombre d'élèves impliqués. Ces travaux sont en général optimistes, prenant la classe comme interlocuteur collectif dont ils décrivent l'activité, en inférant l'apprentissage de chacun. Il y a bien sûr des exceptions, comme l'équipe Éducation et scolarisation (Escol) qui perpétue l'héritage de B. Bernstein et repère les différences de cadrage dans l'activité, les mécanismes de discrimination, sans forcément s'engager dans l'analyse précise du langage attesté dans ce processus. L'approche qualitative sur de petits corpus a pu entrainer le travers d'une interprétation d'exemples choisis comme significatifs d'une dynamique de débat, au détriment d'une évaluation d'apprentissages effectifs. On note cependant une réaction récente pour s'appuyer sur des corpus conséquents, permettant une vue d'ensemble.. Revient aussi, assez timidement, le souci d'une évaluation quantitative dans la durée, permettant une vision différenciée des 
évolutions selon les élèves, avec par la force des choses une reprise de critères traditionnels plus simples et robustes, malgré les critiques dont ils avaient fait l'objet (longueur des énoncés, présence de connecteurs, subordonnées) $)^{5}$.

12 Le corolaire a été la spécialisation parallèle des approches linguistiques et psycholinguistiques des difficultés de langage. L'analyse de la langue des élèves, les critères et outils d'évaluation, de remédiation linguistique, moins investis par la didactique (hormis en maternelle), ont été pris en charge par des approches plus techniques et spécialisées. La question des difficultés à l'oral n'a pas fait en didactique du français l'objet d'un investissement comparable à celui des échecs dans l'apprentissage de la lecture. Cette tendance me semble poser question, à l'heure où les enquêtes internationales font prendre conscience que le problème de notre système scolaire n'est pas tant d'améliorer l'excellence que de combattre l'écart qui s'accroit entre les bons et ceux qui ne le sont pas. La réflexion autour des composantes d'un socle commun semble peu stimulante pour la didactique du français, et cela interroge sur ses priorités et ses modes de fonctionnement.

\section{Les savoirs sur l'oral, leur rapport avec l'oral comme objet didactique}

13 Quelles évolutions concernant la définition de l'objet de travail ? Cerne-t-on mieux ses niveaux, les priorités d'un enseignement? Tous les articles à toutes les périodes soulignent la polysémie du terme, les savoirs et pratiques hétérogènes qu'il recouvre, d'où la confusion des objectifs et des critères, le flottement des formulations. Un des chantiers de la période a été, sinon de stabiliser une définition homogène de l'objet de travail, de tenter du moins une clarification, en sériant des niveaux ou des facettes selon lesquels son apprentissage peut être envisagé, et leurs relations. Cela peut être des structurations binaires (objet/moyen d'enseignement, pour apprendre/à apprendre, formel non formel) ou selon un triple niveau (social, cognitif, linguistique). Cet effort a eu le mérite de clarifier les domaines d'objectifs et d'expliciter les points de clivages entre définitions.

\subsection{Apports et avancées}

Un premier acquis a été de débarrasser l'approche de l'oral de fausses représentations, en cernant mieux sa réalité. Cette meilleure connaissance de la langue parlée est une réelle avancée, malgré des effets qui peuvent être ambigus. Sur la période antérieure, je renvoie à la synthèse de 1999, simplifiant ici quelques traits où l'évolution a été sensible : perspective contrastive opposant ordres oral et scriptural (pour la langue, le récit..), notions et exercices transposés du français langue étrangère (FLE - communication, niveaux de langue...), méconnaissance de la réalité linguistique de l'oral dans le monde scolaire (la notion de niveaux de langue par exemple ne repose pas sur une analyse d'oraux parlés). L'inventaire en 1999 disait que des ressources linguistiques devenues disponibles avaient permis une connaissance plus juste de la langue parlée, de voir par exemple que des traits d'oralité communs à tous renvoient aux conditions de l'énonciation à l'oral. La diffusion d'analyses conversationnelles de diverses obédiences (E. Roulet, logique illocutoire), les travaux de F. François sur les dialogues d'enfants ou d'autres équipes (Groupe de recherche sur les interactions conversationnelles - GRIC - 
puis Interactions, corpus, apprentissages, représenattions - ICAR - à Lyon entre autres) sur les échanges scolaires ont permis l'élargissement aux polylogues, fourni des outils d'analyse d'autres conduites que le récit monologué, popularisé des notions comme modes d'enchainement, reformulation, coénonciation.. Ces descriptions ont en partie levé des préjugés, déplacé critères de jugement et objectifs d'amélioration (par exemple par prise de conscience de l'importance des relations sémantiques et pragmatiques pour juger de la cohérence).

15 La généralisation des enregistrements et transcriptions est une autre avancée. Les évolutions technologiques (enregistrement audio et vidéo, logiciels de traitement de données) ont multiplié les corpus attestés permettant un travail fin. Le livre pionnier de C. Blanche-Benveniste en 1987 avait initié une réflexion explicite sur la transcription: quelle transcription pour ne pas aplatir l'oral, en dégager la structure ? Le film a apporté des éléments nouveaux, la multimodalité des échanges notamment, amenant aussi une réflexion sur ce qu'on produit en filmant (Guernier, Durand-Guerrier \& Sautot, 2007). Certaines équipes (Groupe aixois de recherches en syntaxe - GARS -, ICAR) travaillent depuis longtemps sur de grands corpus permettant une étude quantitative, le projet Visa vise la mise en commun de corpus et d'outils, le $\mathrm{n}^{\circ} 147-148$ de Pratiques en a analysé les méthodes et les problèmes. Même si dans les conditions ordinaires de la recherche didactique, il s'agit plutôt de petits corpus étudiés de façon qualitative, l'usage d'enregistrements, de transcriptions s'est répandu dans les articles de recherche et les groupes de travail. Enseignants et formateurs se sont habitués à voir de l'oral, admettre ses particularités, moins se focaliser sur les traits d'oralité. Chez les chercheurs, cette période semble avoir marqué un déplacement dans les méthodes, faisant de la transcription d'extraits de classe une sorte de norme pour l'écriture didactique, témoignant du souci de prendre en compte les pratiques langagières attestées par lesquelles prennent corps les innovations - référence au travail concret en classe qui était minoritaire en didactique à la période précédente. Le problème de ce qu'on demande à ces extraits, si ce n'est pas un simple effet de réel, reste une question épistémologique importante.

16 L'articulation de l'oral et de l'écrit est un point sur lequel il y a eu de réelles avancées. Cette question a été longtemps pensée de façon binaire, en opposant deux entités homogènes : pour faire de la langue parlée un objet d'étude, les linguistes se sont centrés au début sur ce qui était spécifique (dialogue, langage d'action, langages émergents ou déviants), et en anthropologie, la diffusion biaisée des premiers travaux de J. Goody, en sociologie de B. Lahire a accrédité l'idée d'un "grand partage", cautionnant la dichotomie traditionnelle entre ordres oral (immédiateté, affectivité, absence de distance critique) et scriptural (distance réflexive, raisonnement symbolique). Ces dichotomies, persistantes dans l'opinion scolaire, ont été remises en cause à la période suivante, sur le plan théorique, en linguistique comme en anthropologie, et par l'observation des situations sociales et scolaires qui montre que l'oral intervient dans des formes mixtes, articulé à des activités d'écrit. J.-F. Halté récuse cette dichotomie lors d'un colloque sur l'oral, des travaux didactiques isolés ont dès les années 90 étudié ces situations d'intrication entre oral et écrit et les effets réciproques que cela produit ${ }^{6}$. C'est une piste didactique importante pour désenclaver et valoriser l'oral dans ses contributions au travail en français. On ne compte plus les articles sur l'interprétation de textes ou d'albums qui produisent des extraits parlés d'élèves, mais sans que la perspective didactique soit centrée sur le travail langagier : l'oral est un peu partout, dans les débats 
notamment, mais comme transparent, cette présence n'amenant pas forcément une attention précise à sa réalité et aux problèmes de formulation à dépasser.

\subsection{Les problèmes corolaires aux avancées}

17 Cette avancée des savoirs sur l'oral a permis d'ajuster les objectifs et les jugements évaluatifs, de légitimer des conduites orales plus variées que le récit, mode privilégié du travail et de l'évaluation jusque-là, mais a aussi suscité plusieurs problèmes.

18 La meilleure attention à l'oral et les moyens de l'enregistrer ont complexifié son appréhension et rendu plus multiforme sa réalité. Le langage en situation s'articule à des actions partagées, des signes supra-verbaux (intonation), non verbaux (regards, gestes), articulation fondamentale dans l'acquisition du langage et son développement, comme J.M. Coletta (2000) l'a montré pour les gestes. Les travaux intégrant la multimodalité des échanges se sont multipliés (par exemple Filletaz \& Schubauer-Leoni , 2008), renouvelant l'approche des modes de thématisation, d'établissement de la cohérence ou d'ajustement des interprétations. En même temps cela entraine une prolifération de la description, risque connu des ethnométhodologues, et la centration sur de petits fragments. Un problème méthodologique fondamental auquel s'est confrontée la période a donc été celui de l'échelle et du grain d'analyse, comme dit M. Foucault, des corpus mobilisés, pour tenter d'articuler des microanalyses à des portées plus grandes. d'objectifs et d'indicateurs multiples a décentré la recherche d'objectifs spécifiquement langagiers. Jugée restrictive dans ses formulations antérieures de la complexité ou de la qualité linguistiques, la référence linguistique s'est diluée dans l'étude des interactions ou des discours. La période précédente avait travaillé à dégager des critères linguistiques pour évaluer la richesse ou les blocages dans le dialogue, des fils rouges pour l'organisation de parcours d'apprentissage. Il y a des points de consensus sur les indices de développement langagier, les travaux sur l'acquisition continuent à apporter des connaissances, surtout pour les débuts du langage, mais il n'est pas sûr que la recherche ait beaucoup progressé sur le développement linguistique d'élèves plus âgés. Les travaux qui permettraient de se faire une idée précise, linguistiquement fondée des acquis, des points de langue problématiques ou mal maitrisés ne sont pas légion. Cette relative désaffection des analyses portant sur les ressources de langue et usages attestés me semble valoir aussi pour l'écrit, où les travaux articulant les dimensions discursive et linguistique dans l'analyse de l'énonciation écrite d'élèves ne sont pas si nombreux.

Enfin, la tension entre description et prescription n'est pas résolue par l'accroissement des connaissances. L'histoire de la didactique de l'oral montre à quel point elle est chargée d'enjeux liés à des postures militantes, bien que se référant à des travaux théoriques récusant la prescription. Même si les descriptions de linguistes ou d'analystes de la conversation ont induit une tolérance vis-à-vis de fonctionnements jugés autrefois fautifs et relativisé des critères bien ancrés, toute didactique, surtout celle du langage, est confrontée au problème de la norme, en ce qu'elle vise l'enrichissement de pratiques en fonction de critères qu'elle doit expliciter. Pourtant la réflexion explicite sur la norme et les variations, vivante dans les années $70^{7}$, ne se poursuit plus que dans quelques équipes, comme Dysola-linguistique (Dylis) à Rouen, en relation avec le plurilinguisme, la didactique comme discipline de recherche revendiquant davantage une fonction de production de connaissances sur les dialogues, les pratiques scolaires, d'où le glissement 
vers des travaux descriptifs sur les interactions. De ce fait, la définition de normes (objectifs, critères d'évaluation) est laissée à d'autres (inspecteurs, manuels). En même temps, les descriptions mobilisent malgré elles des valeurs, même en refusant toute mission prescriptive, ne serait-ce que dans le choix des exemples, et l'axiologie est présente. Une autre rançon de la complexification est la difficulté de prendre en charge la question des progressions, sauf pour certains secteurs plus balisés comme le récit. En l'état actuel, peu d'éléments permettent de fonder une mesure de progrès dans la pratique orale de tous les élèves, tant elle pose de problèmes méthodologiques et théoriques. Faute d'analyse des effets, la recherche sur l'oral se trouve donc souvent dans une position ambigüe, hésitant à assumer la formulation de normes pour orienter l'action des enseignants (définir les éléments d'un "socle ", les critères d'une progression), mais pouvant difficilement ne pas en véhiculer. On dispose cependant de ressources pour démêler des niveaux de normes, des paramètres de variation selon les fonctions de la verbalisation, les référents et les situations, les analyses d'échanges en situation de travail ayant élargi la conception de ce que peut être un langage efficient, producteur de sens. L'avancée a donc été de s'attacher à clarifier objectifs et critères pour juger du développement de conduites orales, organiser des tâches et prendre une mesure des progrès, avec la difficulté que ces normes sont contextualisées, intriquées à d'autres niveaux de normes, qui ne relèvent pas des mêmes temporalités d'apprentissage et peuvent entrer en conflit (Nonnon, 2005). La période a tenté d'expliciter ce que voudrait dire enseigner dans le cas de l'oral, et - c'est une évolution - de davantage confronter les choix didactiques à l'épreuve de la pratique et de ses contraintes.

\section{Les tâtonnements autour de l'enseignabilité et des modèles de l'enseignement}

21 La question l'oral peut-il être constitué en objet d'enseignement ? est récurrente ${ }^{8}$. Que signifie enseigner dans ce cas ? La structuration du champ sur ce point a longtemps été présentée de façon dichotomique. Qu'en est-il maintenant?

Sur l'objet lui-même, il s'est établi explicitement certains points de consensus, sur lesquels il faut insister pour ne pas céder à des oppositions schématiques :

- l'enjeu de l'enseignement scolaire de l'oral est l'entrée progressive dans un oral public, différent de la conversation, permettant de structurer l'expérience, d'élaborer des connaissances, des jugements, d'ouvrir la communication à d'autres enjeux que la connivence ;

- ce développement s'effectue à travers des activités langagières plus larges que les petites unités (genres discursifs, conduites langagières) ;

- le travail oral suppose des dispositifs comportant des situations contrôlées mais ouvertes pour susciter ces conduites, et des éléments de progression. Il est nécessaire d'évaluer des progrès imputables à l'apprentissage, ce qui implique un enseignement dans la durée.

Mais la question de l'enseignabilité de l'oral engage des définitions différentes des activités langagières à développer, de l'enseignement et de l'apprentissage, des missions de la recherche. Le terme de genre, par exemple, en vogue à cette période, correspond malgré la référence commune à $M$. Bakhtine à des acceptions différentes, renvoyant soit aux genres normés pratiqués dans l'univers social (ou à l'image qu'on s'en fait) voire aux genres formels de la rhétorique, soit comme chez F. François aux configurations souples 
mais régulières mises en œuvre dans les conduites langagières (raconter, expliquer, exposer, résumer, qui articulent un rapport à l'interlocuteur, un positionnement de soi, une forme de structuration du référent, un type de cohérence - François, 1993).

Face aux principes évoqués et aux contraintes constitutives d'un enseignement explicite (clarification des apprentissages visés, possibilité d'évaluation des acquis) on peut faire le choix de la modélisation scolaire d'un répertoire de discours sociaux stylisés, dont les facettes et les normes sont l'objet d'un travail explicite et structuré dans le temps, ce qui a été l'orientation de l'équipe de Genève. On lui a opposé une approche intégrée, dont la visée était de dégager dans les situations variées de verbalisation en classe, les ressorts pouvant en faire l'occasion d'un travail langagier, de les exploiter systématiquement, par l'explicitation de variables didactiques suscitant des seuils dans la verbalisation et l'organisation des tâches (Repères, $n^{\circ} 24-25,2003$ ). Les problèmes communs qui soustendent ces choix interdisent une approche polémique caricaturale, et l'évolution des travaux, en essayant de mettre les propositions didactiques à l'épreuve d'une analyse de leur mise en œuvre en classe, a amené des deux côtés nuances et enseignements. Le travail d'évaluation de M. Wirthner sur la mise en œuvre par des enseignants d'une séquence didactique genevoise est significatif de cette évolution : elle essaie de dégager la logique selon laquelle chacun s'approprie des éléments de l'outil fourni par les didacticiens et de comprendre comment leur réticences à la particularité du genre proposé peut s'expliquer par leur conception de ce qui est important (dans ce cas la capacité générale, transférable de hiérarchiser les informations). De même l'observation de pratiques intégrées montre qu'il n'est pas facile de gérer à la fois des objectifs de langage et des objectifs disciplinaires, de mener le dialogue de façon différenciée dans une classe hétérogène et de construire des interactions génératrices d'apprentissages pour tous. Il est apparu que la critique de P. Rayou et E. Bautier sur les «classes bavardes" n'est pas infondée, le temps laissé aux conversations pouvant être discriminant et le travail qui s'y effectue peu lisible pour certains, et qu'on n'apprend en discutant qu'à certaines conditions. Cette mise à l'épreuve est un signe de maturité, mais suppose une durée longue dans la recherche, tenant compte des apories ou des questions de validité révélées par les recherches précédentes, pour ne pas toujours repartir sur les mêmes projets.

Ces deux pôles stylisent en fait des questions de fond qui se posent aussi pour d'autres apprentissages en français, notamment l'écriture. La première est la tension entre un apprentissage sériel, abordant successivement des formes de discours spécifiques dans leurs caractéristiques particulières (les genres, souvent travaillés comme autrefois les types de textes), ou un travail autour d'axes communs de développement fondant la dynamique de conduites langagières diverses (exposition d'une situation, dramatisation, intégration de l'interlocuteur, évaluation au sens de W. Labov). Le fondement est différent, même si le travail en classe peut engager des pratiques intermédiaires (marquer dans un apprentissage sériel ce qui peut être transféré à d'autres genres; dans l'approche intégrée pratiquer une progressivité et une évaluation critériée dans l'apprentissage de genres scolaires par exemple).

La deuxième question touche aux modèles de référence fondant l'enseignement et l'évaluation. Dans une approche déductive à partir de modèles stylisés, l'éloignement des pratiques discursives réelles, plus souples et mobilisant des critères pragmatiques et sémantiques plus que formels, fait que le temps passé sur des artéfacts risque de produire des effets limités au niveau du transfert, la plupart des pratiques à développer échappant 
à ce répertoire restreint. Dans la seconde perspective, le travail d'élucidation est à faire, moins par déduction de normes génériques que par élucidation et affinement de jugements évaluatifs en situation, puisque la qualité d'une production discursive n'est pas pensée comme adéquation à des normes externes, mais comme rapport instable entre différents niveaux du discours et interaction de critères hétérogènes. Cette structuration contextualisée des tâches et des exigences a été poursuivie, surtout pour la maternelle, plus pour le récit que pour d'autres conduites. Mais elle est très exigeante et il faudrait pour assoir visées et critères accroitre des savoirs sur les usages effectifs et ce qui pourrait être attendu aux différents âges

Cependant des savoirs (linguistiques, socio ou psycholinguistiques), des références (l'autorité de L. Vygotski entre autres) ne suffisent pas pour changer les normes et les pratiques de ceux qu'on souhaite éclairer ou former. Pour s'inscrire dans une interaction dynamique, la recherche didactique doit prendre en compte la façon dont les enseignants arrivent à gérer ces normes diverses, souvent en tension, qui sous-tendent l'oral de l'école. Faute d'en tenir compte, elle ne peut faire que des propositions décalées.

\section{Le rapport de la didactique à l'analyse du travail}

Les aléas de la didactique de l'oral tiennent aussi à ce que sa pratique est directement liée au présent concret du travail en classe, dont on ne peut l'abstraire. Le travail d'enseignement passe en grande partie par l'engagement de l'enseignant dans les interactions orales avec ses élèves, et c'est à travers leur analyse que beaucoup de chercheurs ont cherché à appréhender ce travail. L'observation et la catégorisation des interventions enseignantes et de leurs fonctions dans la gestion du dialogue didactique bénéficient depuis longtemps de typologies et d'outils, chez M. Postic ou M. Altet par exemple. Ces descriptions de l'activité enseignante en général ont été tardivement reprises en didactique du français et se sont développées à la période récente, dans une perspective de formation, en référence à l'analyse du travail et des gestes professionnels. S'ils reposent sur une analyse d'interactions orales, les types d'interventions dégagés relèvent souvent de la gestion générale du temps scolaire, du groupe, des avancées de l'activité en cours, plus que de visées didactiques sur des apprentissages langagiers ou des objets de travail spécifiques à la discipline. Par ailleurs la référence à l'ergonomie est incomplète si elle ne prend pas en compte l'économie du travail enseignant comme travail, donc la logique et le point de vue des enseignants, même s'ils contrarient à première vue celui du chercheur.

Pour les enseignants, la pratique de l'oral est couteuse dans les conditions ordinaires et des contextes scolaires qui sont souvent loin de fonctionner comme des communautés harmonieuses et homogènes centrées sur l'acquisition de savoirs. L'investissement en temps, par sa lourdeur, peut leur sembler tenir peu compte de la pluralité de contraintes et visées qu'ils essaient de concilier, que soulignent toutes les études sur le travail enseignant. P. Perrenoud (1994) avait formalisé quelques dilemmes spécifiques de la gestion de la parole en classe, tenant à l'interférence de normes intériorisées par les enseignants pour ce domaine de leur activité et au calcul de risques face à une plus large place donnée à la parole des élèves. C'est en les prenant au sérieux qu'une didactique de l'oral a élaboré sinon ses réponses, du moins les ressources qu'elle propose aux enseignants, et que les chercheurs doivent peser leur analyse de la gestion du dialogue scolaire chez ceux qu'ils observent. La méconnaissance de ces conditions ergonomiques 
amène des propositions séduisantes mais peu opératoires, sinon inaudibles. Les travaux en didactique ont donc eu à affronter des questions, théoriques et pratiques, relatives au travail de l'enseignant quand il conçoit et gère les situations d'oral.

La question du nombre est la première contrainte et la difficulté majeure. La gestion d'un groupe nombreux dans les contraintes de l'école (temps, programmes) induit des conduites d'encadrement qui font facilement l'objet de descriptions peu compréhensives de la part des chercheurs (validations trop rapides par exemple). Pour les enseignants, prendre conscience de ce raidissement peut être un moyen de le contrôler pour permettre aux élèves une prise de parole développée et non une simple participation. Mais ce problème du nombre questionne aussi le chercheur, qui doit admettre que la régulation de polylogues ne peut s'appréhender sur le mode de la conversation ou de l'étayage mère-enfant, et comprendre les réticences d'enseignants sur l'économie du temps scolaire, en évitant de confondre l'activité collective qu'il observe avec les apprentissages, qui relèvent de l'appropriation par chacun. Cette prudence n'est pas toujours respectée dans les descriptions de récits en commun ou de débats, nombreuses dans la période récente, tentées de conclure rapidement à des apprentissages à partir d'éventuelles avancées dans l'activité collective, ce qui tronque l'analyse vygotskienne souvent invoquée. On voit les inventions méthodologiques qui seraient nécessaires pour fonder l'évaluation en termes d'apprentissages, ce à quoi s'attèlent quelques travaux récents. La situation de l'enseignant devant gérer l'oral en classe est justement dans cette tension. Il doit résister à ce que G. Sensevy appelle la «différenciation didactique passive », la propension naturelle d'un enseignement efficace à accroitre la dispersion des résultats, laissant les plus avancés profiter le plus des situations didactiques: l'appel systématique à la parole des élèves comporte ce risque d'une capitalisation des bénéfices et d'une prise de pouvoir par ceux qui ont le plus d'acquis. Ce problème d'équité, crucial pour l'oral, vaut aussi pour les activités de lecture et d'écriture, et la sophistication des objectifs et des recherches ne lui donne pas toujours sa vraie place. Il est fondamental pour la didactique de le prendre en compte, si tant est, comme dit G. Sensevy, que « la détermination d'efficacité-équité peut constituer in fine une des raisons d'être de la didactique » (Sensevy, Mercier, 2007).

La contrainte du temps, liée à l'apprentissage, pose aussi problème aux enseignants et aux didacticiens. L'oral, c'est banal de le dire, prend un temps collectif qu'il faut rendre utile aux yeux de tous, et le temps scolaire est compté. La répétition, la régularité sont indispensables pour qu'il y ait apprentissage et évaluation d'une progression, sur des objectifs ciblés, pour chacun - pas seulement pour ceux qui font avancer le temps didactique. Cela suppose de réfléchir aux variables des tâches proposées et à la progression de seuils dans la durée. C'est surtout pour la maternelle, pour le récit, que ce travail s'est continué. Pour le chercheur aussi cette question est cruciale, l'obligeant à admettre que son temps (celui de l'observation, l'enregistrement, l'analyse d'une activité donnée) est différent du temps du travail scolaire et de l'apprentissage, et qu'il ne peut extrapoler légèrement de l'un à l'autre. Un certain nombre de travaux récents marque de ce point de vue une prise de conscience, l'effort d'articuler plusieurs échelles temporelles et d'inscrire des observations ciblées dans une durée longue, en construisant des outils de formalisation pour cela. L'analyse du travail implique aussi que l'investissement soit rapporté à ce qu'il produit, ce qui pose la question de la mesure des évolutions de tous, difficile comme on l'a vu, dès qu'on sort d'un réinvestissement immédiat, et rejoint le critère de l'efficience. On voit combien la prise en compte de cette dimension (le cout 
pour l'enseignant en relation avec les bénéfices constatés) questionne le positionnement de la didactique du français, ses liens avec la formation et les pratiques scolaires.

\section{Conclusion} questionnent la didactique dans ses missions, ses conditions de légitimité face aux
tensions de la pratique, sa définition épistémologique. On mesure ce qui au cours de son histoire a été posé dans la connaissance des usages et des pratiques orales, la formalisation d'indicateurs et de méthodes d'analyse, l'évolution des problématiques normatives, mais aussi ce qui manque encore pour fonder de façon valide des progressions, fournir des points de repère sur l'évolution et le développement de conduites discursives selon les âges, selon diverses variables. L'histoire montre ainsi des continuités de préoccupations, une relative cumulation des savoirs, des changements de focale et des avancées, mais aussi des filiations non explicitées, des défauts de mémoire, des recommencements, parfois des piétinements.

Ceci étant, le travail sur l'oral n'est pas actuellement un secteur florissant de la didactique du français. La continuité de ce travail bute sur des difficultés qui touchent au cœur de la discipline : difficultés méthodologiques, liées à la question du cout des observations en classe, de leur durée, qui renvoie au problème des conditions de validité des conclusions ; questions épistémologiques, relatives au statut des modèles, des régularités génériques, au type de généralité à construire quand on travaille sur des activités contextualisées, en partie imprévisibles, également à la façon dont une discipline à visée scientifique portant sur des pratiques normatives peut penser sa propre dimension normative. Comment faire qu'une plus grande lucidité n'amène pas un désenchantement, une désaffection, ou le détour dans des régions moins difficiles de la recherche ? La confrontation aux difficultés suscite me semble-t-il des indices de maturité : une plus grande conscience des effets produits par le recueil de données, le renoncement aux dichotomies et oppositions frontales de théories par une prise en compte plus nuancée de la réalité de la parole et de l'enseignement, une attention plus grande aux conditions de validité et d'acceptabilité des généralisations et des prescriptions, une réflexion sur les unités d'analyse et les outils pour prendre en compte les questions de temporalité, plus de modestie par rapport au point de vue des enseignants.

Surtout, la question de l'oral rappelle obstinément ce noyau central des visées et des tensions de la didactique du français, si stimulant dans les années 70 et les premiers moments de Pratiques, mais dont on peut penser que les évolutions récentes l'ont plutôt mis à l'arrière-plan : la recherche des objectifs et des conditions pour que les discussions, comme les autres pratiques en français, soient l'occasion de progrès effectifs pour tous et n'accentuent pas les écarts. La mise en veilleuse en didactique du problème de l'oral n'exclut pas que les tensions sociales et l'inquiétude sur les missions de l'école n'imposent de nouveau son urgence, et il est important d'assumer et de faire fructifier cette mémoire. Cette référence à la dimension sociale et démocratique de la didactique du français, si vive dans le premier projet de Pratiques, n'est pas un péché de jeunesse ou une ringardise dont on sourit, mais il faut beaucoup de travail encore pour qu'elle soit plus qu'une incantation. Je finirai par une phrase qui m'a frappée, visitant la veille de cette intervention l'exposition Leiris au centre Pompidou de Metz. À propos de son adhésion à la révolution cubaine avec son ami Wifredo Lam, il écrit dans Frêle bruit : «À aucun prix il 
ne faut qu'un jour vienne où j'aurais honte de moi en pensant à la révolution cubaine. C'est une des pires taches de sang: renoncer à une foi ou la dévitaliser, parce qu'il est trop dur d'avoir à se reprocher de ne pas être à la hauteur ».

\section{BIBLIOGRAPHIE}

AURIAC-SLUSARCZYK, E. (dir.) (2013), Apprendre et former : la dimension langagière. Clermont-Ferrand : Presses universitaires Blaise Pascal.

BACHMANN, C. (1977). « "Il les a dit devant nous il n'avait pas eu peur..." Analyse de conversation ». Pratiques 17, p. 77-99.

BLANCHE-BENVENISTE, C. \& JEANJEAN, C. (1987). Le français parlé: transcription et édition. Paris : Didier. CHISS, J.-L. (2002). Le couple oral/écrit et la tension entre communicatif et cognitif. Colloque de la Desco. Paris. : Didactiques de l'oral.

CICUREL, F. (2011). «De l'analyse des interactions en classe de langue à l'agir professoral : une recherche entre linguistique interactionnelle, didactique et théories de l'action ». Pratiques 149-150, p. 41-55. En ligne : http://pratiques.revues.org/1693.

COLETTA, J.-M. (2000). « La prise en compte de la multimodalité de la parole dans la description et analyse des conduites langagières ». Communication et organisation 18. En ligne : https:// communicationorganisation.revues.org/2427.

DELCAMBRE, I. (2002). « L'inscription graphique au cours d'un travail de groupes ». Pratiques 115-116, p. 93-106.

Denizot, N., LAPARRA, M., MAQUAIRE, M. \& NONNON, É. (200). « Table ronde : L'oral au lycée. Table ronde organisée et mise en texte par Isabelle Delcambre ». Pratiques 107-108, p. 229-242.

FILLETAZ, L. \& SCHUBAUER-LEONI, M.-L. (dirs) (2008). Processus interactionnels et situations éducatives. Bruxelles : De Boeck.

FRANÇOIS, D. (1977). « Traits spécifiques d'oralité et pédagogie ». Pratiques 17, p. 31-52.

- (1980). «Dialogue, discussion et argumentation au début de la scolarité ». Pratiques 28, p. 83-94.

- (1993). Pratiques de l'oral Paris : Nathan.

GARCIA, C. (1980). «Argumenter à l'oral : de la discussion au débat ». Pratiques 28, p. 95-124.

GUERNIER, M.-C., DURAND-GUERRIER, V. \& SAUTOT, J.-P. (dirs) (2007). Interactions verbales didactiques et apprentissages. Besançon : Presses universitaires de Franche-Comté.

HALTÉ, J.-F. (1983). « De la langue à la communication dans l'école ». Pratiques 40, p. 3-16.

- (1992). La didactique du français. Paris : Presses universitaires de France.

- (dir.) (1993). Inter-actions : l'interaction, actualités de la recherche et enjeux didactiques. Metz : Centre d'analyse syntaxique de l'Université de Metz.

- (2006). « Entre enseignement et acquisition : problèmes didactiques en apprentissage du langage ». Mélanges Crapel 29, p. 13-28. 
MOUCHON, J. \& FILlol, F. (1977). « Problématique et enjeu de l'enseignement de l'oral ». Pratiques

17, p. 3-8.

MULLER MIRZA, N. \& BUTY, C. (dirs) (2015). L'argumentation dans les contextes d'éducation. Berne : Peter Lang.

NONNON, É. (1999). «L'enseignement de l'oral et les interactions verbales en classe : champs de référence et problématiques ». Revue française de pédagogie 129, 1, p. 87-131. En ligne : http:// www.persee.fr/doc/rfp_0556-7807_1999_num_129_1_1067.

- (2002). « Des interactions entre oral et écrit : notes, canevas, traces écrites et leurs usages dans la pratique orale ». Pratiques 115-116, p. 73-92.

- (2005). « Entre description et prescription, l'institution de l'objet : qu'évalue-t-on quand on évalue l'oral ? Repères 31, p. 161-188.

- (2011). «L'histoire de la didactique de l'oral, un observatoire de questions vives de la didactique du français? ». Pratiques 149-150, p. 184-206. En ligne : http://

pratiques.revues.org/1739.

PERRENOUD, P. (1994). « La communication en classe : onze dilemmes ». Cahiers pédagogiques 326, p. 13-18.

SENSEVY, G. \& MERCIER, A. (2007). Agir ensemble : l'action didactique conjoints. Rennes : Presses universitaires de Rennes.

\section{NOTES}

1. Le Francais aujourd'hui $n^{\circ} 39$ (1977), Pratiques $n^{\circ} 17$ (1977), le Centre de recherche de l'éducation spécialisée et de l'adaptation scolaire (CRESAS), équipes de D. et F. François à Paris V, E. Espéret, M. Brossard à Bordeaux, B. Gardin à Rouen...

2. Dans Pratiques $n^{\circ} 28$ (1980), article de F. François sur l'argumentation émergente, précédemment cité, est significatif de cette visée.

3. $\mathrm{N}^{\circ} 17$ de Repères («L'oral pour apprendre », 1998), $\mathrm{n}^{\circ}$ 103-104 de Pratiques («Interactions et apprentissages ", 1999, avec, dans ces recueils, l'idée de décalages dans l'interprétation de la situation chez les protagonistes et d'inégalités dans le collectif), travaux de l'équipe de Bordeaux. 4. Parmi les travaux récents, on trouve E. Auriac-Slusarczyk (2013) et N. Muller Mirza \& C. Buty (2015).

5. Par exemple chez P. Péroz pour le récit en maternelle, ou E. Auriac-Slusarczyk lors d'ateliers philosophiques en primaire (mais ce n'est pas une recherche de didactique du français).

6. Par exemple $n^{\circ} 81$ des Études de linguistique appliquée (1991) : « l'écrit dans l'oral „. Le n 115-116 de Pratiques comporte deux articles sur cette intrication. Le n 24-25 de Repères, «Enseigner l'oral » (2003), inventorie dans une visée didactique diverses formes d'articulation entre oral et écrit dans les activités de classe.

7. Question abordée dans Pratiques 17 ; voir aussi Le Français aujourd'hui 101 (1993) : « Normes et pratiques de l'oral ».

8. Le titre du n' 39-40 d'Enjeux (1997), "vers une didactique de l'oral ?», comportait un point d'interrogation, comme un titre de J.-F Halté en 2002 : «Pourquoi faut-il oser l'oral ?». La forme affirmative L'oral s'enseigne, ou infinitive: Enseigner l'oral (Repères $\mathrm{n}^{\circ} 24-25$ ) a toujours une dimension combative et exploratoire. 


\section{RÉSUMÉS}

Bien que les savoirs sur la langue parlée et les interactions se soient beaucoup développés, la recherche didactique sur l'enseignement de l'oral a connu malgré ses enjeux une histoire à éclipses. L'évolution des discours depuis 40 ans est significative à la fois des spécificités de cet objet de travail, et de questions et tendances plus générales de la didactique du français face aux problèmes qui se posent à elle, qu'elle a assumés de façon différente, ou contournés plus ou moins selon les périodes. La question de l'oral rappelle avec insistance ces problèmes: les inégalités de langage et les moyens d'éviter les discriminations dans les activités de français, la place du travail sur la langue, la généricité et la contextualisation des normes et des modèles, les rapports entre description et prescription, la prise en compte de l'économie du travail scolaire dans les analyses et les incitations.

Although knowledge on the spoken language and interactions developed much, didactic research on oral teaching has had, in spite of its issues, an ecliptic history. Since 40 years, discourses evolution on this subject has been both significant for the very specific object of work, and broad for questions and trends associated to French didactic according to the issues it arises, assumes in different ways or circumvents more or less according to the periods. The question of oral teaching insistently reminds these deep problems: language inequalities and means of avoiding discriminations in the activities of French; importance of the work on the language; generic and contextualised standards and models; relationships between description and prescriptions; the economy of schoolwork in the analyses and incentives.

\section{INDEX}

Mots-clés : didactique du français, langue parlée, interactions verbales, normes et variations, modèles de discours, travail enseignant.

Keywords : French didactics, spoken language, verbal interaction, standard and variations, models of speech, teaching work

\section{AUTEUR}

\section{ÉLISABETH NONNON}

Université de Lille 3, CIREL, EA 4354, F-59000, France 